evaluation of candidate genes/SNPs. The availability of GWAS should, theoretically, solve the second problem. We have very few GEWIS (genome-wide interaction study) on occupational exposures and they suffer even more than candidate-gene studies from sample size and also from lack of available studies for replication.

Results Apart from exposures, GEWIS may help identify new diseases genes since many may only have an effect in combination with exposure. In addition GEWIS should provide a much more complete evaluation of specific pathways, e.g. oxidative stress. The use of a specific pathway-based approach is still valuable if based on biological knowledge. Contrary to major advances brought by molecular epidemiology, studies on geneenvironment interactions have proven to be complex and with few well established findings.

Conclusions I will discuss reasons for this, discuss recent changes from the use of genome-wide analyses and compare with earlier approaches based on evaluations of interactions of occupational exposures with few candidate genes. I will provide examples from a recent GEWIS on occupational asthma.

\section{THE CONTRIBUTION OF MOLECULAR EPIDEMIOLOGY TO STUDYING OCCUPATIONAL DISEASE: SOME ACCOMPLISHMENTS TO DATE AND OPPORTUNITIES FOR THE FUTURE}

${ }^{1}$ Nat Rothman, ${ }^{2}$ Roel Vermeulen, 'Qing Lan. ${ }^{1}$ National Cancer Institute, Bethesda, Md, USA; ${ }^{2}$ IRAS Utrecht University, Utrecht, The Netherlands

\subsection{6/oemed-2014-102362.391}

Objectives There are several contributions that molecular epidemiology can make to the study of occupational disease.

Method These include 1) enhancing the ability to study doseresponse relationships; 2) evaluating the biological plausibility that an exposure may be related to an adverse outcome; and 3) providing insight into the underlying biological mechanism of an established or suspected exposure-disease relationship. These goals can overlap at times.

Results There are multiple examples where molecular epidemiology studies have contributed important information about occupational exposures, including instances where such studies contributed data that played a role in determining if a given exposure was causally related to disease. Historically, these studies have complimented classic epidemiological investigations.

Conclusions There are multiple examples where molecular epidemiology studies have contributed important information about occupational exposures, including instances where such studies contributed data that played a role in determining if a given exposure was causally related to disease. Historically, these studies have complimented classic epidemiological investigations.

\section{CAN EXPOSURE-RESPONSE CURVES BASED ON MOLECULAR EPIDEMIOLOGY DATA INFORM THE EXPOSURE-RESPONSE CURVE?}

${ }^{1}$ Roel Vermeulen, ${ }^{2}$ Qing Lan, 'Jelle Vlaanderen, 'Lutzen Portengen, ${ }^{2}$ Nat Rothman. ${ }^{1} /$ ras Utrecht University, Utrecht, The Netherlands; ${ }^{2}$ National Cancer Institute, Bethesda, Md, USA

10.1136/oemed-2014-102362.392
Objectives Derivation of the exposure-response curve at low (occupational) exposures is often troubled by the fact that within epidemiological investigations power to discern the exposureresponse curve (ERC) at low exposure levels is often limited. Conversely, we often observe non-linear exposure-response curves at the higher end of the exposure range which amongst others may be due to metabolic saturation.

Method Derivation of the exposure-response curve at low (occupational) exposures is often troubled by the fact that within epidemiological investigations power to discern the exposureresponse curve (ERC) at low exposure levels is often limited. Conversely, we often observe non-linear exposure-response curves at the higher end of the exposure range which amongst others may be due to metabolic saturation.

Results Studies on benzene exposed occupational populations have indicated 1) non-linear production of reactive metabolites at low levels of exposure; 2) non-linear production of benzeneoxide adducts; and 3) non-linear associations between benzene and hematoxicity. This is of particular interest as there have been indications of a possible non-linear association between benzene and leukaemia in epidemiological studies.

Conclusions The evidence on a molecular and clinical level may provide evidence for a possible non-linear association between benzene and leukaemia and provides promise that molecular data can directly be integrated in epidemiological risk analyses to inform ERCs.

\section{IS THE FUTURE IN EPIGENETICS?}

Andrea Baccarelli. Mark and Catherine Winkler Associate Professor of Environmental Epigenetics, Exposure, Epidemiology and RiskProgram, Laboratory of Human Environmental Epigenetics, Harvard School of Public Health,Office-LandmarkCenter, Room 415 E West, P. O. Box 15677401 Park Drive, Lab-Building 1, Room B-12 665 Huntington Avenue, Boston, MA, USA

\subsection{6/oemed-2014-102362.393}

\section{Objectives Is the future in epigenetics?}

Epigenetics investigates heritable changes in gene expression that occur without changes in DNA sequence. Several epigenetic mechanisms, including DNA methylation and histone modifications, can change genome function under exogenous influence. Results obtained from animal models indicate that in utero or early-life environmental exposures produce effects that can be inherited transgenerationally and are accompanied by epigenetic alterations. The search for human equivalents of the epigenetic mechanisms identified in animal models is in progress. I will present evidence from human environmental and occupational studies indicating that epigenetic alterations may mediate effects caused by toxic exposures. In these investigations, we have shown that exposures, including air pollution, lead, arsenic, nickel, and PAHs, are associated with altered methylation of human repetitive elements or genes. In recent preliminary studies, we have shown alterations of histone modifications and miRNAs in subjects exposed to metal-rich airborne particles. I will present original data demonstrating that altered DNA methylation in blood and other tissues is associated with potentially related disease, such as cardiovascular disease and asthma. On the basis of current evidence, I will propose possible models for the interplay between toxicants and the human epigenome.

Method Please see above.

Results Please see above.

Conclusions Please see above. 